\title{
Sub-Band-Based Transmission for Mode-Multiplexed Optical Systems
}

\author{
Lailson F. dos Santos ${ }^{1}$, Filipe M. Ferreira ${ }^{2}$, Darli A. A. Mello ${ }^{1}$ \\ ${ }^{1}$ School of Electrical and Computer Engineering, State University of Campinas (UNICAMP), Campinas, Brazil \\ ${ }^{2}$ Aston Institute of Photonic Technologies, Aston University, Birmingham, B4 7ET, UK \\ Tel: +55 193521 3808, e-mail: darli@decom.fee.unicamp.br
}

\begin{abstract}
Mode-multiplexed optical transmission is subject to mode coupling and potentially large differential mode delays. In most recent implementations, these effects are compensated for at the receiver by complex adaptive MIMO equalizers. Although frequency-domain MIMO equalization requires a moderate complexity compared to time-domain equalization, the long required FFTs may face implementation issues and yield a relatively slow response to dynamic effects. In this paper, we evaluate an alternate transceiver architecture based on sub-band partitioning, implemented by filter banks, which enables concurrent time-domain equalization. The performance of sub-band and single-carrier schemes are compared using Monte-Carlo simulations.
\end{abstract}

Keywords: sub-band partitioning, space-division multiplexing, MIMO equalization.

\section{INTRODUCTION}

Space-Division Multiplexing (SDM) has been accepted as the only transmission technique able to cope with the exponential traffic growth experienced in several segments of data networks, specially in inter and intra data center interconnects. Besides parallel transmission of signals in bundles of single-mode fibers (SMFs), multicore fibers (MCFs) and multimode fibers (MMFs) are important candidate technologies for the SDM transmission media. Signals conveyed by MCFs with uncoupled cores are easy to couple and switch, but require careful crosstalk management. On the other hand, MMFs use typically multiple-input multiple-output (MIMO) processing to separate the signals multiplexed over the multiple orthogonal modes. The complexity of such MIMO equalizers is specially challenging in mode-multiplexed systems with large differential mode delays (DMD) and low or intermediate mode-coupling levels. Although in frequency-domain equalization (FDE) the computational complexity scales with the logarithm of the delay spread, the application-specific integrated circuit (ASIC) area reserved for the fast Fourier transforms (FFTs) may become excessively large [1], and the equalizer may react slowly to dynamic effects.

A promising alternative to avoid large FFT sizes is to split the spectrum into smaller sub-bands and carry out source separation and deconvolution in parallel for the individual sub-channels [2]. This idea has been validated in conventional polarization multiplexed systems (PM) transmission using a computationally-efficient structure with polyphase filters (called in the paper a twice-underdecimated filter bank) [3]. The technique has been applied to mitigate chromatic dispersion (CD) [4] and non-linear effects [5]. Polyphase filter bank architectures have also been employed in the context of MIMO equalization for MMFs in [6], where the twiceunderdecimated filter bank architecture was adapted to achieve fast convergence in an orthogonal frequency division multiplexing (OFDM) system with weakly coupled spatial channels. Alternatively, sub-band partitioning in the optical domain has been reported in [7] to mitigate DMD in MMFs and coupled-core MCFs.

In this paper, we investigate the performance of the multi sub-band (MSB) architecture in MIMO SDM equalization with digitally-generated subcarriers. We focus on a transceiver design based on Nyquist-shaped subcarriers generated by a common prototype filter, as proposed in [3][4]. Compared with OFDM, this architecture exhibits the advantage of avoiding bandwidth consumption due to cyclic prefixes. We compare the performance of a conventional single-carrier (SC) system with that of a MSB architecture at the same data rate, using Monte-Carlo simulations. The remainder of this paper is organized as follows. Section 2 presents the architecture of the MSB system. Section 3 describes the simulation setup, including the optical channel model. Section 4 discusses the simulation results. Lastly, Section 5 concludes the paper.

\section{MULTI SUB-BAND (MSB) SYSTEM}

Figure 1 shows the transmitter diagram of the MSB system investigated in this paper. This structure is replicated for each of the $\mathrm{Nm}$ spatial modes. The data generated by a binary source are first mapped into a complex constellation. The modulated complex sequence is then parallelized into an odd number of streams $(N s b)$. The DFT-based polyphase filter bank is implemented with $N s b+1$ data streams, where the stream in position $(N s b+1) / 2+1$ contains zeros to avoid edge effects [4]. A root-raised cosine (RRC) filter is used as prototype. The transmitted data in each sub-band operate on a symbol rate of $R s b=1 / T s b=R s c / N s b$, where $R s c$ is the rate for the single-carrier case. After that, the signals are serialized, pre-distorted, and fed into a complex MachZehnder (MZ) for electro-optical conversion. 


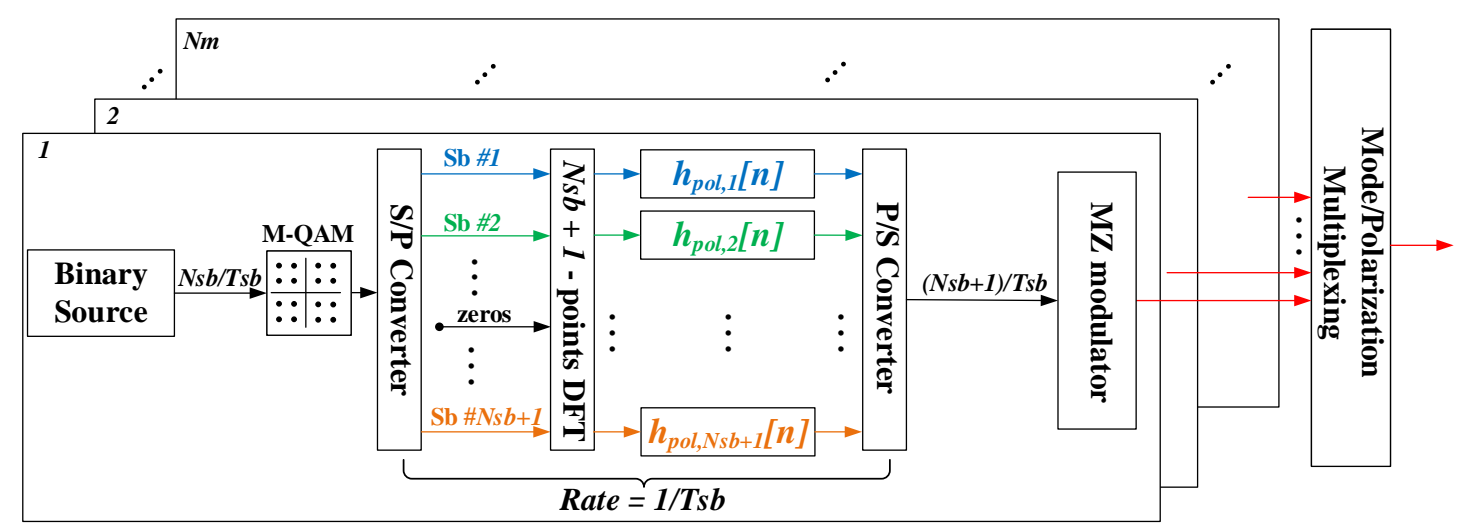

Figure 1. Multi sub-band transmitter.

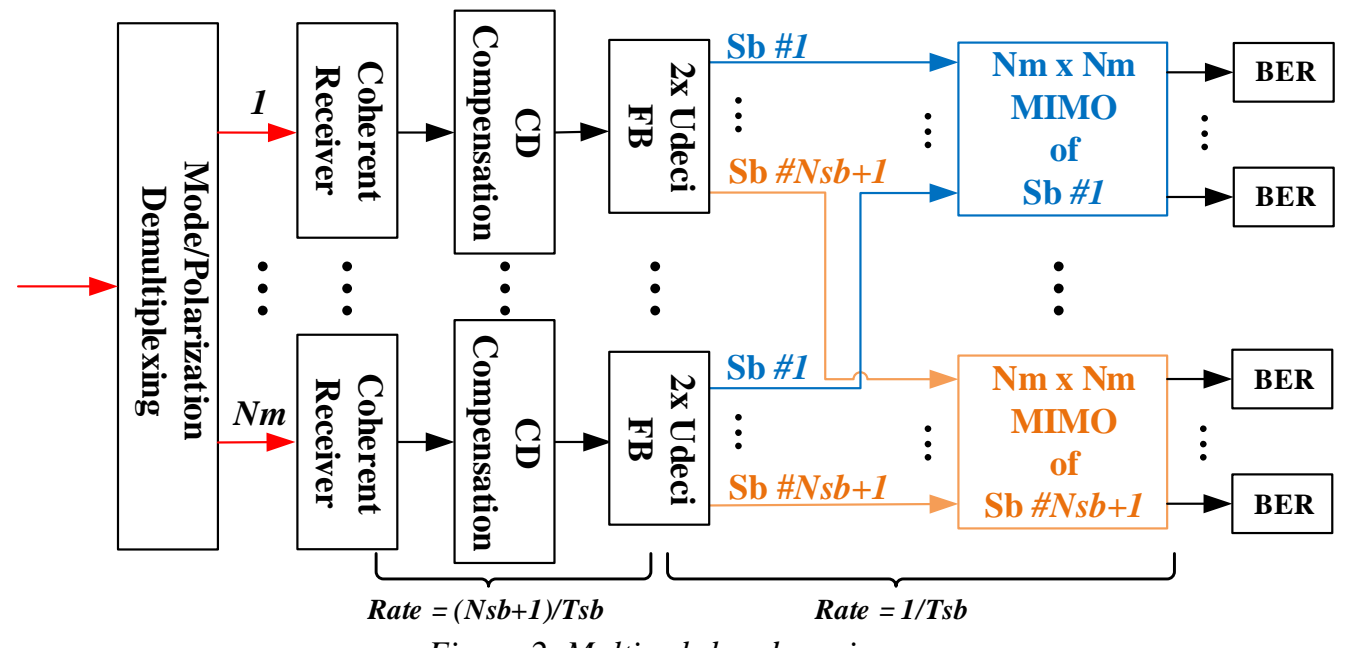

Figure 2. Multi sub-band receiver.

The sub-band receiver structure is shown in Fig. 2. After optical mode and polarization demultiplexing, the $\mathrm{Nm}$ signals are converted into the electric domain and are sampled at a rate of $(N s b+1) / T s b$. Notice that the MSB architecture requires nearly half the number of samples compared to the SC counterpart, which typically operates with 2 samples per symbol (SPS). Each modal signal is then subject to chromatic dispersion compensation using a frequency-domain static filter [8]. The signal then follows to the twice-underdecimated filter bank (2x Udeci FB) based on polyphase filters that are matched to the transmitted waveforms [3]. Although the DSP architecture receives a signal with 1 SPS in each sub-band, the 2x Udeci FB block produces 2 SPS for subsequent equalization. After that, the signals are sent to $N s b \mathrm{Nm} \times \mathrm{Nm}$ MIMO adaptive equalizers, one for each sub-band. The structure of the MIMO adaptive filters is a generalization of the $2 \times 2$ MIMO butterfly equalizer for PM signals [8].

\section{SIMULATION SETUP}

The simulations for both the MSB and SC systems were based on the 16-QAM modulation format at $256 \mathrm{~Gb} / \mathrm{s}$, an electric filter bandwidth of $16 \mathrm{GHz}$ and $1550 \mathrm{~nm}$ wavelength. All adaptive equalizers implemented timedomain equalization (TDE). Although for long channels FDE offers a considerably lower complexity, the purpose of the paper is to investigate TDE in the context of sub-band systems, in a way to avoid long FFTs.

The SC transmission setup implemented Nyquist-shaped pulses (RRC shaping filter with 513 coefficients and 0.01 roll-off factor) at a symbol rate of $R s c=32$ Gbaud. An initial sequence of 200,000 symbols was used for supervising the convergence of the $12 \times 12$ MIMO equalizer (corresponding to 6 linearly polarized (LP) modes and the respective polarization modes), which was updated by the least-mean squares (LMS) algorithm. After this training phase, the filter coefficients remained static, as the simulated channel did not vary over time. The bit error rate (BER) was counted by the average value obtained over 50,000 symbols in each spatial mode.

The symbol rate for the MSB simulation corresponds to Rsc divided by the number of sub-bands, so as to achieve the same bit rate. The RRC prototype filter was implemented with 0.01 roll-off factor and length of the polyphase filters $\left(h_{p o l}\right)$ was set to 128 taps. A training sequence of 30,000 symbols was used for convergence of the $N s b$ 12x12 MIMO adaptive equalizers, implemented individually in each sub-band. After training, the filter coefficients remained static. The BER was counted by averaging the results obtained over $(25000 / N s b)$ symbols transmitted in each sub-band, i.e., 25,000 symbols per spatial mode. The simulation run twice for each point. The 
transceiver architecture simulation was evaluated in back-to-back, and with a $100 \mathrm{~km}$ optical fiber model containing $-30 \mathrm{~dB} / \mathrm{km}$ crosstalk, $20 \mathrm{ps} / \mathrm{km}$ DMD. The chromatic dispersion (CD) vector is $(22.18,21.55,22.15$, $22.15,21.84,21.84) \mathrm{ps} /(\mathrm{km} \cdot \mathrm{nm})$ and group delay vector $(-1.12,-11.26,-2.53,-2.53,8.73,8.73) \mathrm{ps} / \mathrm{km}$ for modes (LP01, LP02, LP11a, LP11b, LP21a, LP21b), respectively.

The simulated optical channel model [9] describes the fiber imperfections responsible for mode coupling as random fluctuations of the core centre position, given by: $\varepsilon(x, y, z)=\varepsilon_{p}(x+d x(z), y+d y(z), z)$, where $\varepsilon$ is the perturbed permittivity, $\varepsilon_{p}$ is the ideal relative permittivity, $d x$ and $d y$ are the random displacement of the abscissa and ordinate coordinates, respectively. The model divides the fiber in multiple sections, each with a constant random displacement of the core centre position. Therefore, each section has constant coupling coefficients allowing to use semi-analytical solutions [10] for the set of differential equations describing the mode coupling. Finally, the mode coupling strength is set by varying the fibre displacement, as show in [11].

\section{RESULTS}

Figure 3 shows the convergence time of SC and MSB systems with crosstalk and DMD. The filter lengths and step sizes were optimized for every condition. The SC case required approximately 120,000 symbols to converge (see Fig. 3a), which is significantly higher than in usual PM systems. With MSB the number of required symbols reduced significantly, reaching an order of approximately 20,000 symbols. Although the MSB system required a lower number of symbols to reach convergence, the overall convergence time should take into account the fact that the symbol period is also scaled by the number of sub-bands. The convergence time (in microseconds) for the MSB and SC systems is shown in Fig. 3b, and shows no evident relationship with the number of sub-bands. Both figures indicate a systematic BER penalty in the MSB transceiver compared with the SC option after convergence. This penalty has been also observed in previous works with MSB systems, and can be attributed to interference among neighbouring sub-bands [4].
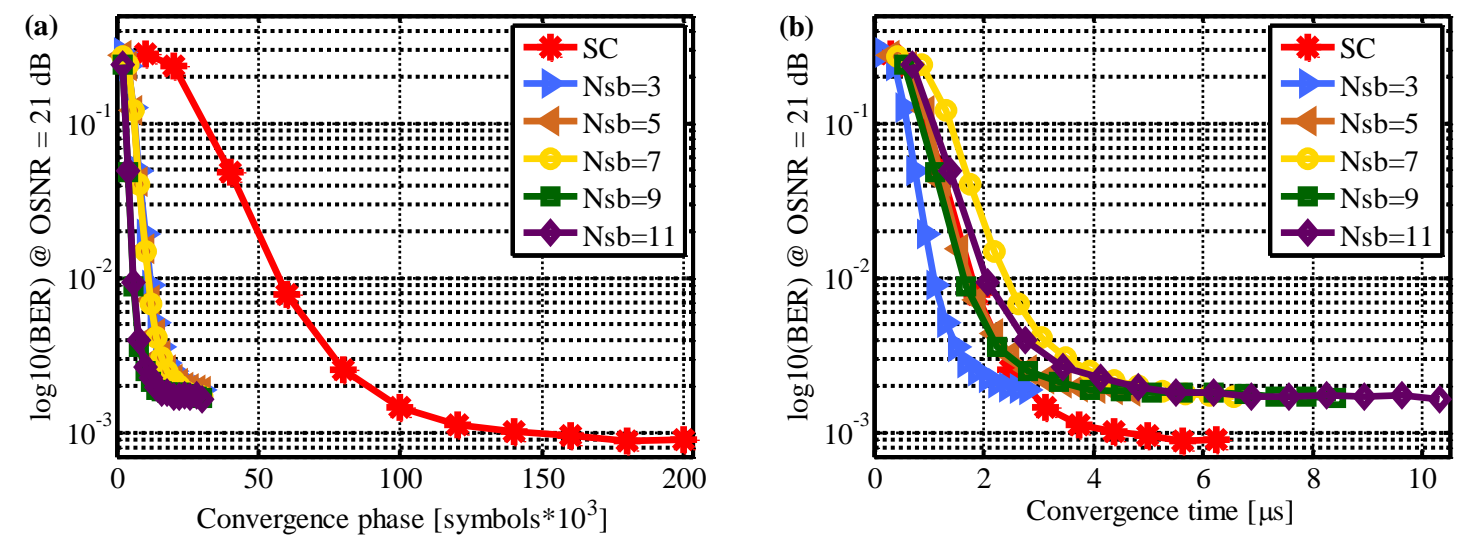

Figure 3. Convergence period for OSNR $=21 \mathrm{~dB}$ of MSB and SC systems with crosstalk and DMD.

Figure 4 shows the optical signal to noise ratio (OSNR) required to achieve a BER of 4e-3, versus number of equalizer filter taps, for different configurations. The simulation searched for the required OSNR within a resolution of $0.1 \mathrm{~dB}$. The back-to-back performance of SC and MSB systems is presented in Fig. 4a. The simulations reveal an OSNR penalty of approximately $0.5 \mathrm{~dB}$ for the MSB system compared with the SC, because of inter-sub-band interference. The SC performance for configurations with DMD and uncompensated $\mathrm{CD}$ are shown in Fig. 4b. The DMD alone increases the required filter length to approximately 250 taps. When $\mathrm{CD}$ is left uncompensated, the minimum filter length raises to 280 taps. The MSB system subject to DMD is shown in Fig. 4c. The filter length required for a satisfactory performance varied from 30 taps (11 sub-bands) to 70 taps ( 3 sub-bands). The results for the case where CD is left uncompensated is shown in Fig. $4 \mathrm{~d}$, and indicates that the impact of $\mathrm{CD}$ on the equalizer filter length is negligible.

\section{CONCLUSIONS}

We evaluated MSB transmission using filter banks for space-division multiplexed systems based on few-mode fibers. Sub-bands allow to parallelize MIMO processing avoiding excessively long FFTs required in typical SC frequency-domain equalizers. The results indicated that, although MSB requires a lower number of symbols for convergence, the overall convergence time may be lower, equivalent or even higher than the SC option, depending on the implemented number of sub-bands. As expected, the MSB architecture requires considerably shorter filters and allow parallel signal processing. As a drawback, the MSB architecture exhibited a systematic penalty of $0.5 \mathrm{~dB}$ compared with the SC case. This may be caused by linear interference between adjacent subbands, as observed in previous papers. Further work should evaluate the SC and MSB performance under timevarying channels. 
(a)

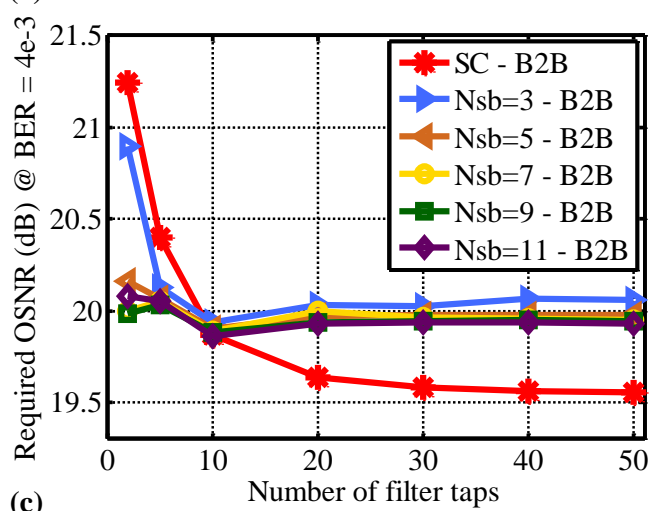

(c)

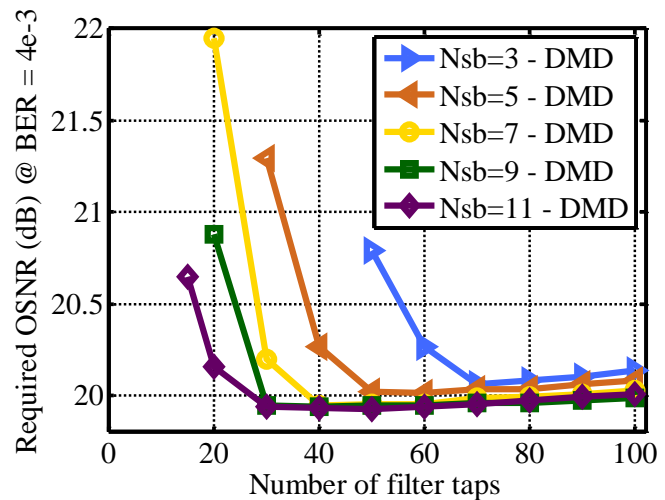

(b)

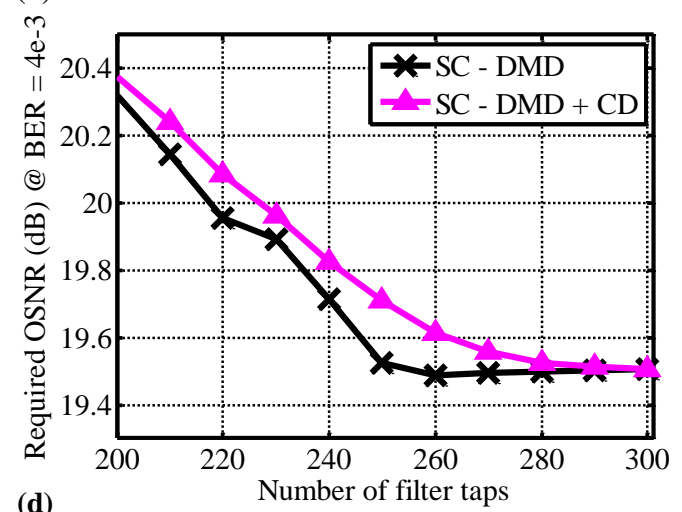

(d)

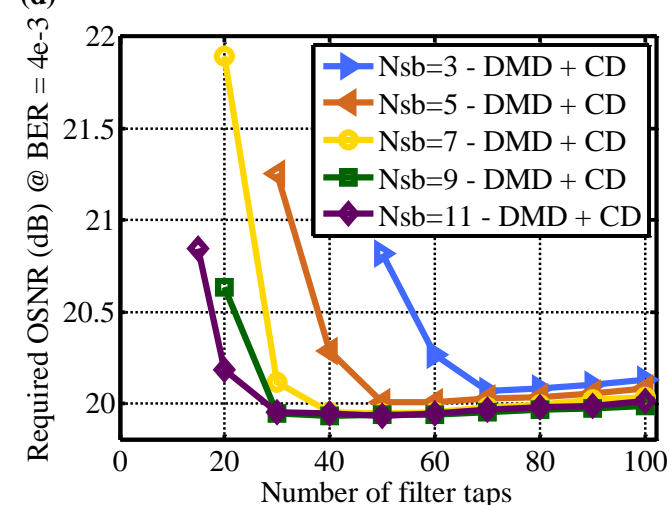

Figure 4. (a) Back-to-back transmission for MSB and SC systems. (b) Optical channel with crosstalk, DMD and compensated and uncompensated CD, for the SC system. (c) Optical channel with crosstalk and DMD for MSB system. (d) Optical channel with crosstalk, DMD, and uncompensated CD for the MSB system.

\section{ACKNOWLEDGEMENTS}

The authors would like to thank Capes for the financial support.

\section{REFERENCES}

[1] N. Bai et al:: Experimental demonstration of adaptive frequency-domain equalization for mode-division multiplexed transmission, in Proc. OFC/NFOEC 2013, Anaheim, CA, Mar. 2013, pp. 1-3.

[2] K. P. Ho: Subband equaliser for chromatic dispersion of optical fibre, in Electronics Letters, vol. 45, no. 24, pp. 1224-1226, Nov. 2009.

[3] M. Nazarathy and A. Tolmachev: Subbanded DSP architectures based on underdecimated filter banks for coherent OFDM receivers: overview and recent advances, in IEEE Signal Processing Mag., vol. 31, no. 2, pp. 70-81, Mar. 2014.

[4] M. Malekiha, I. Tselniker, and D. V. Plant: Chromatic dispersion mitigation in long-haul fiber-optic communication networks by sub-band partitioning, Opt. Express 23, pp. 32654-32663 (2015).

[5] M. Qiu et al:: Subcarrier multiplexing using DACs for fiber nonlinearity mitigation in coherent optical communication systems, in Proc. OFC 2014, San Francisco, CA, Mar. 2014, pp. 1-3.

[6] M. Nazarathy and A. Tolmachev: Digital sub-banding for coherent single-mode and spatial division multiplexed fiber systems, OECC/ACOFT 2014, Melbourne, VIC, July 2014, pp. 374-376.

[7] T. Mizuno et al.: 12-core $\times 3$-mode dense space division multiplexed transmission over $40 \mathrm{~km}$ employing multi-carrier signals with parallel MIMO equalization, in Proc. OFC 2014, San Francisco, CA, Mar. 2014, pp. 1-3.

[8] S. J. Savory: Digital filters for coherent optical receivers, Opt. Express vol.16, pp. 804-817, 2008.

[9] F. Ferreira et al.: Nonlinear semi-analytical model for simulation of few-mode fiber transmission, IEEE Photon. Technol. Lett., vol. 24, no. 4, pp. 240-242, Feb. 2012.

[10] F. Ferreira, P. Monteiro, and H. Silva: Semi-analytical model for linear modal coupling in few-mode fiber transmission, in Proc. ICTON 2012, July 2012, p. Th.A1.5.

[11] F. Ferreira et al.: Few-mode fibre group-delays with intermediate coupling, in Proc ECOC 2015, Valencia, Sept. 2015, p. Th.1.6.1. 